\title{
Zwangsstörungen
}

\section{Eine Domäne der Psychotherapie}

\author{
Zwangsstörungen zählen mit einer Lebenszeitprävalenz von etwa 2\% [1] \\ zwar nicht zu den häufigsten, aber zu den hartnäckigsten psychischen \\ Erkrankungen. Betroffene leiden nicht selten unter schweren Beeinträch- \\ tigungen des Berufs- und Privatlebens. Oft werden Zwangsstörungen \\ erst spät entdeckt - meist im Zusammenhang mit Begleiterkrankungen \\ wie Angst, Alkoholismus oder affektiven Störungen.
}

Zi wangsgedanken oder Zwangshandlungen, die sich meist zwischen dem 20. und 25. Lebensjahr manifestieren, sind außerordentlich vielgestaltig und persistieren ohne Therapie bei der Hälfte der Betroffenen lebenslang. Mit $40 \%$ fast ebenso häufig sind schwere Beeinträchtigungen des Berufs- und Privatlebens. Hinzu kommt, dass Zwangsstörungen aus Scham oder fehlender korrekter Diagnose oft erst spät entdeckt werden meist im Gefolge von Begleiterkrankungen wie Angst, Alkoholismus oder affektiven Störungen, wie Professor Ulrich Voderholzer, Prien am Chiemsee, berichtete. Das diagnostische Screening erleichtern jedoch schon wenige Fragen, etwa nach extremer Sauberkeit, häufigen Waschritualen, Kontrollbedürfnissen, beunruhigenden Gedanken sowie nach erhöhtem Zeitaufwand für tägliche Verrichtungen.

Das Spektrum der Zwangsstörungen, im neuen DSM-5 als eigene Kategorie von den Angststörungen abgekoppelt, reicht von der durch Ängstlichkeit und Risikovermeidung charakterisierten Zwanghaftigkeit bis zu Impulskontrollstörungen, bei denen risikosuchendes Verhalten wie Kleptomanie oder pathologisches Kaufverhalten im Vordergrund stehen.

Ätiologisch kommt psychologischen Faktoren wie Traumata oder Belastungen wie Verlustängste oder Einsamkeitsempfindungen sowie neurobiologischen Charakteristika größere Bedeutung zu als genetischen Ursachen [2], wenngleich das Erkrankungsrisiko naher Verwandter von Zwangspatienten etwa vier- bis sechsfach erhöht ist.

Neurobiologisch findet sich oft eine Störung fronto-striato-thalamischer Regelkreise, bei der die Balance der Neurotransmitter Serotonin, Dopamin und Glutaminsäure gestört ist [2]. Für eine Schädigung neuronaler Strukturen als Ursache der Zwangsstörung spricht laut Voderholzer auch, dass sich bei $20 \%$ der

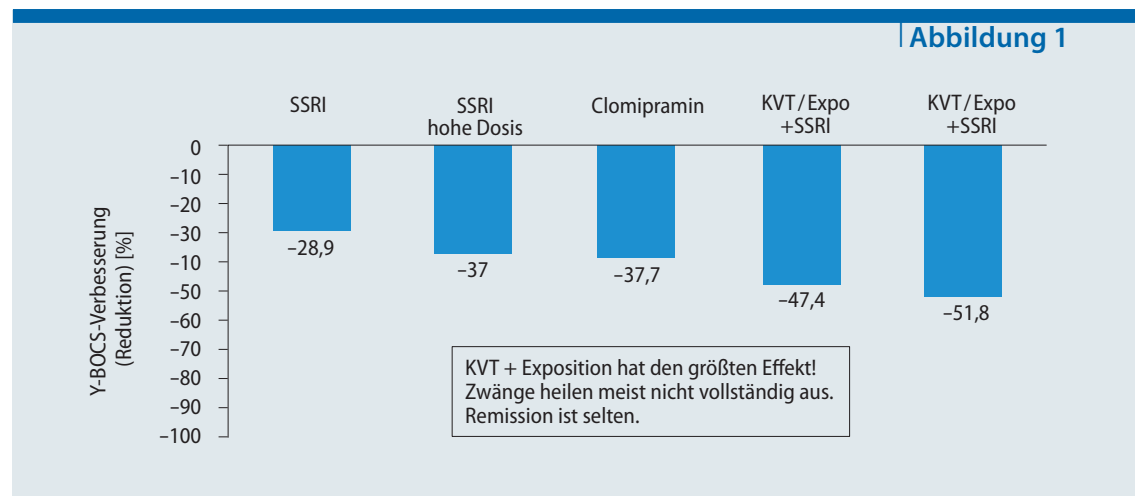

Durchschnittliche Abnahme der Symptome bei Zwangsstörungen in großen Therapiestudien, Y-BOCS-Verbesserung (Reduktion) [nach 5].
Patienten gegen Basalganglien gerichtete Antikörper nachweisen lassen [3].

Zwangserkrankungen sind primär eine Domäne der Psychotherapie. Therapie der ersten Wahl ist die Kombination aus kognitiver Verhaltenstherapie (KVT) mit der Exposition gegen aversive Stimuli [4]. Letztere dürfe nie unterlassen werden, sollte graduiert erfolgen und zumindest in der Anfangsphase von einem Therapeuten begleitet werden, empfahl Voderholzer. Die - mit Responderraten von 60-70\% und einer mindestens $35 \%$ igen Symptomreduktion - sehr effektive Maßnahme lässt sich in der Wirksamkeit durch Kombination mit selektiven Serotonin-Wiederaufnahmehemmern (SSRI) oder mit Clomipramin noch etwas steigern. Umgekehrt wirken die Medikamente alleine weniger stark als die KVT. Allerdings tritt die Wirkung der Pharmaka frühestens nach vier Wochen ein und das Rückfallrisiko beträgt bei alleiniger Gabe $80-90 \%$. Eine neue Option bei Nichtansprechen auf SSRI ist das atypische Neuroleptikum Aripiprazol [5]. Es bietet zudem den Vorteil der fehlenden Gewichtszunahme, kann allerdings Unruhe und Schlafstörungen verursachen. Aripiprazol induziert bei Patienten mit Schizophrenie außerdem deutlich weniger Zwangssymptome als die dort bisher verwendeten Substanzen Clozapin und Olanzapin [6]. Auch achtsamkeitsbasierte Psychotherapie zusätzlich zur Exposition ist nach einer ersten kontrollierten Studie signifikant wirksam [7].

Andreas Häckel

1. Voderholzer U et al. Nervenarzt 2011; 82: 273-4

2. Karch S, Pogarell O. Nervenarzt 2011; 82: 299-307

3. Nicholson TR et al. Brit J Psychiatry 2012; 200: $381-6$

4. Voderholzer, Hohagen (Hrg.) Therapie psychischer Erkrankungen, Urban \& Fischer, 8. Aufl. 2012

5. Sayyah $\mathrm{M}$ et al. Depression and Anxiety 2012; 29: 850-4

6. Schirmbeck F et al. J Psychopharmacol 2013; 27 (4): 349-57

7. Hertenstein E et al. BMC Psychiatry 2013; 12: 185 